\title{
Criteria for the definition of Pituitary Tumor Centers of Excellence (PTCOE): A Pituitary Society Statement
}

\author{
Felipe F. Casanueva ${ }^{1} \cdot$ Ariel L. Barkan $^{2} \cdot$ Michael Buchfelder $^{3} \cdot$ Anne Klibanski $^{4} \cdot$ Edward R. Laws $^{5}$.

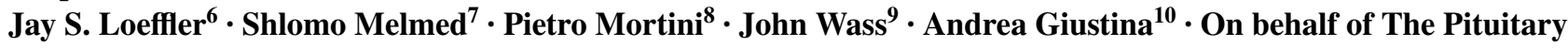 \\ Society, Expert Group on Pituitary Tumors
}

Published online: 7 September 2017

(C) The Author(s) 2017. This article is an open access publication

\begin{abstract}
Introduction With the goal of generate uniform criteria among centers dealing with pituitary tumors and to enhance patient care, the Pituitary Society decided to generate criteria for developing Pituitary Tumors Centers of Excellence (PTCOE).

Methods To develop that task, a group of ten experts served as a Task Force and through two years of iterative work an initial draft was elaborated. This draft was discussed, modified and finally approved by the Board of Directors of the Pituitary Society. Such document was presented and debated at a specific session of the Congress of the Pituitary Society, Orlando 2017, and suggestions were incorporated. Finally the document was distributed to a large group of global experts that introduced further modifications with final endorsement.

Results After five years of iterative work a document with the ideal criteria for a PTCOE is presented.
\end{abstract}

Collaborators for The Pituitary Society, Expert Group on Pituitary Tumors.

\section{Felipe F. Casanueva}

endocrine@usc.es

1 Division of Endocrinology, Santiago de Compostela University, Santiago de Compostela, Spain

2 Division of Endocrinology, University of Michigan Health System, Ann Arbor, MI, USA

3 Department of Neurosurgery, University Hospital Erlangen, Erlangen, Germany

4 Neuroendocrine Unit, Massachusetts General Hospital, Boston, MA, USA

5 Department of Neurosurgery, Brigham \& Women's Hospital, Boston, MA, USA
Conclusions Acknowledging that very few centers in the world, if any, likely fulfill the requirements here presented, the document may be a tool to guide improvements of care delivery to patients with pituitary disorders. All these criteria must be accommodated to the regulations and organization of Health of a given country.

Keywords Acromegaly $\cdot$ Cushing's disease ·

Thyrotropinomas · Gonadotropins · Transsphenoidal surgery $\cdot$ Pituitary radiotherapy

\section{Introduction}

The pursuit of excellence is a continuous endeavour for health professionals and is increasingly sought by society and health administrators alike. It is widely accepted that only experts in a field may provide the best standard of care to patients. In this context, pituitary tumors are more frequent than previously thought $[1,2]$ and present a significant challenge for diagnosis and management. There is a widely

6 Department of Radiation Oncology, Massachusetts General Hospital, Boston, MA, USA

7 Cedars-Sinai Medical Center, Los Angeles, CA, USA

8 Department of Neurosurgery, San Raffaele University Health Institute Milan, Milan, Italy

9 Oxford Centre for Diabetes, Endocrinology and Metabolism, Churchill Hospital, Oxford, UK

10 Division of Endocrinology, San Raffaele University Hospital, Milan, Italy 
held consensus endorsed by publications in the field, indicating that the best care for these patients is provided by an interdisciplinary team composed of dedicated endocrinologists and experienced pituitary surgeons working in collaboration $[3,4]$. Such a core team needs to be supported by a collaborative environment of specialists in other areas, such as neuroradiology, neuropathology, radiation oncology, neuro-ophthalmology, otorhinolaryngology, plus trained nursing [4].

The goals of that team should include: (1) early detection of the tumor; (2) establishing the diagnosis; (3) determination of the most suitable treatment, which can either be observation; surgical, medical or radiotherapy; (4) if surgical, removing the pituitary mass while preserving the normal pituitary tissue and nearby structures; (5) the surgical or medical treatments working alone or concomitantly must eliminate hormonal hypersecretion and/or its effects; (6) prevention of tumor recurrence; and (7) recognizing and caring for the acute and delayed complications of the disease, especially hypopituitarism. The final goal is the elimination or at least reduction of the excess morbidity and mortality associated with the tumor and hypersecretion syndrome as well as treatment of accompanying pituitary hormone insufficiencies [3-5]. For many patients, this requires a program of care, including medical therapy, surgery and radiation therapy, in addition with long-term follow-up.

\section{The need for Pituitary Tumor Centers of Excellence (PTCOE)}

The concept of a Center of Excellence has been promulgated in various fields of medicine to address public and professional concerns regarding the quality of care of a given group of patients. This concept has been found to be useful primarily in activities involving multidisciplinary teams and more often in teams that are composed of experts in surgical techniques and medical treatments [6-8]. In some centers treating pituitary tumors, highlevel interdisciplinary groups are already developed and effectively function, but in most cases, these groups are self-appointed structures without formal acceptance by hospital managers, or health authorities, no by their colleagues, and there are no formal definitions of the criteria needed to be considered truly "excellent" [4]. If there are no explicit requirements for achieving a degree of excellence, patients' outcomes cannot be accurately measured, and the fulfillment of the previously outlined goals cannot be determined. In addition, the evaluation and accreditation of such structures by external and independent bodies are not performed.
Despite efforts to foster improved adherence to the recommended standards being available, a major barrier to optimal care in patients with pituitary tumors is a delivery system that too often is fragmented, lacks clinical information capabilities, duplicates services, and is not well designed for the delivery of co-ordinated chronic care.

The relationship between quality and outcome has been intuitively affirmed by endocrinologists who have a "preferred pituitary neurosurgeon" to whom they refer patients. However, in a given hospital, efforts by endocrinologists or administrators for example to limit surgical procedures to a single surgeon, may encounter firm opposition by the neurosurgery staff stating that "a graduate in Neurosurgery can perform any intervention". In such situations, surgeons perform all of the activities without sub specialization. In the absence of external guidelines generated and endorsed by authoritative bodies, the status quo is unlikely to change.

A relevant point when dealing with the concept of a PTCOE is performance of scholarly scientific discovery and the transparent communication of results and outcomes. Units formed by a lone endocrinologist or an individual neurosurgeon will have difficulty communicating results to symposia and administrative organizations. This is a crucial point in eliminating "communication bias", i.e., the fact that small groups or groups that perform poorly never present results. Therefore, the literature is full of reports from the more successful groups in the world with the best outcomes and this bias contributes to the fact that there is limited understanding of the true results when evaluating outcomes of new drugs or new surgical approaches. In this context, the implementation and use of disease-specific registries and electronic clinical files may provide a reliable tool with which to communicate unbiased results. As part of their mission, endocrinologists, as well as neurosurgeons and other specialists caring for patients with pituitary disorders, have a duty to make progress in the pituitary sciences, and this is only attainable through teamwork.

Articles indicating the need for Centers of Excellence for pituitary diseases have mostly focused on surgical procedures outcomes [9-15]. Despite convincing arguments, such efforts have not yet been able to change the general surgical practice. On occasion, health or administrative authorities have attempted to define the characteristics of such centers of excellence, but frequently, the main goal was to reduce costs, and these characteristics have not been accepted by the professionals involved in the day-to-day care of the patients. For such reasons, a practical and effective definition of a Pituitary Tumor Center of Excellence (PTCOE) is needed, and the characteristics of such are summarized herein. 
BOX 1. General characteristics of a PTCOE are:

Provide the best care for patients with pituitary tumors and related pathologies

Independent of health authorities, administrations and for-profit organizations

Widely recognized by endocrinologists and pituitary surgeons Aimed to the advancement of pituitary science

Providing adequate patient education and community outreach

Recognized by external national and/or international endocrine and neurosurgical medical societies

Act as training center for residents in the treatment of pituitary pathologies

Based on the characteristics outlined above, we conclude that to provide the best care to patients with pituitary tumors, it is desirable to identify PTCOEs in a given health system area. For a group of endocrinologists who specialize in pituitary disorders and pituitary surgeons participating in such units, it is mandatory to know in an explicit way the requirements and conditions needed to develop such a center and to evaluate its success. These need to be established by an external learned body that may or may not perform the final step of validation of the center.

\section{BOX 2. Mission of the PTCOE}

1. Provide the best standard of care to patients with pituitary tumors and disorders

2. Organize multidisciplinary clinical management

3. Liaision between experienced neurosurgeons and expert neuroendocrinologists

4. Work with the supporting specialties

5. Train fellows in the management of pituitary tumors and related disorders

6. Provide courses, publications and lectures for primary care physicians and other specialists

7. Capture and track clinical data

8. Provide up to date and comprehensive patient information

9. Present results and outcomes to scientific bodies and administrators

10. Support endocrine units located outside the PTCOE

11. Advise health administrators and authorities on specific problems

12. Advance the science and scholarship of pituitary tumors

13. Include tumor data on National or Regional registries

A PTCOE need to envisioned on a "patient centric" organization, as the patient is the core of its mission Patient Networks, engagement activity, family impact, educational platforms, digital infrastructure to facilitate care across primary-secondary health care, and across specialties are essential for a properly focused PTCOE.

\section{Excellence in pituitary surgery}

Although advances in the medical treatment of pituitary tumors over the last decades have been stellar, it is beyond doubt that a PTCOE is dependent on the presence of a dedicated and excellent group devoted to pituitary surgery by the endonasal transsphenoidal or transcranial approaches.

In fact, assuming that modern techniques of imaging, and neuronavigation are available at a given center, the presence of an experienced neurosurgeon capable of performing pituitary microsurgery in a safe and effective manner is mandatory. Pituitary surgery is the most effective procedure for acromegaly, Cushing's disease, TSH-secreting adenomas, resistant prolactinomas and non-functioning pituitary adenomas causing mass effects. It is also effective for pituitary apoplexy, diagnostic uncertainly as to the nature of the lesion and, in the rare cases of pituitary cancer. This approach is also recommended for para-sellar pathologies, such as craniopharyngiomas, Rathke cleft cysts, some chordomas and some skull base meningiomas [9].

BOX 3. Targets of pituitary surgery

1. Eliminate pituitary hypersecretory syndromes

2. Eliminate, reduce or control the tumor mass

3. Preserve the normal pituitary gland function

4. Preserve surrounding neural structures, including the optic apparatus, other

cranial nerves and parasellar vasculature

5. Reduce or eliminate acute complications generated by the tumor

6. Reduce or eliminate the risk of tumor recurrence

Although defining targets for pituitary surgery is relatively easy, defining excellence for an individual pituitary surgeon is considerably more difficult. An excellent neurosurgeon requires a solid knowledge of hypothalamic-pituitary organ physiology and the principles of its endocrine evaluation, plus continuous practice to maintain his/her level of surgical expertise; otherwise, the quality of the surgeon's work may deteriorate.Therefore, an experienced high-level pituitary neurosurgeon requires solid training in basic neurosurgery and continuous practice, and the latter is based on a continuous high workload and demonstrated evidence based outcomes.

The basic knowledge of neurosurgery relies on a residency program that is a sine qua non for a future excellent neurosurgeon. However, in most centers in most countries, the program provides limited experience in transsphenoidal pituitary surgery. This is because most residency training centers perform a small number of transsphenoidal surgeries per year. This situation does not allow the graduate to gather enough experience to be able to practice independently immediately after completing training [9].

Conversely, specific intervention to the pituitary region through an endonasal approach requires a clear understanding of skull base anatomy and the expertise necessary to maneuver instruments and the endoscope in such a narrow surgical space $[16,17]$. In addition, the residency training program for a neurosurgeon is so demanding that graduates have insufficient experience in management of pituitary patients, and 
insufficient interactions with neuroendocrinologists. For these reasons, a graduate who wishes to have a significant pituitary tumor practice in the future should have an additional fellowship at a high-quality center performing a large number of interventions each year [18]. With such a combination of a residency plus a fellowship, the future pituitary tumor surgeon should then have the appropriate education and training.

After the residence, depending on the training opportunities available at a given country, we would recommend either (1) completion of a formal postgraduate fellowship in pituitary surgery, (2) completion of a postgraduate fellowship in skull base or neuro-oncologic surgery at a high volume pituitary center, or (3) completion of postgraduate subspecialty training at a high volume pituitary center.

BOX 4. Basic requirements for excellence for a given neurosurgeon using transsphenoidal procedures

1. Basic residency training in neurosurgery at an accredited center

2. Post-residency fellowship (12-15 months) in an active, high-level pituitary surgery unit, or extensive training in pituitary surgery and pituitary patient management at an established pituitary center

3. Continuous practice in a newly created or previously recognized unit with a high pituitary workload and demonstrated outcomes

4. Contribute to the advancement of Pituitary Science through publications in medical journals, chapters in books and monographics

\section{Pituitary surgery. Outcomes and expertise}

One of the peculiarities of surgery in general, and of pituitary surgery in particular, is that if a superbly trained surgeon does not maintain a high number of operative cases in the following years, the surgeon may lose the capability of performing at an excellent level. Expertise then requires basic training, specific training and continuous practice. For such reasons, several reports have shown that experienced surgeons have better outcomes and lower rates of complications than surgeons with less experience [9, 19-24]. In a US national survey on complications of transsphenoidal surgery conducted on a large number of active neurosurgeons, respondents were divided into three groups depending on number of pituitary surgeries performed along their life-long experience. The morbidity and mortality was lower for the surgeons with more interventions, and the rate of complications was lower. Despite that, the most experienced surgeons witnessed more severe complications in absolute terms, related to the high numbers of operative procedures, and also because they operate on the most complicated cases [24]. Additionally, in cases of surgical reoperation when the first surgery is complicated or fails, surgical experience is crucial for obtaining optimal results [25-28].

After having a neurosurgeon with the correct training during residency who afterwards performs a fellowship at a high level center, what makes the difference between experienced and less experienced surgeons? The answer is the number of procedures performed by the surgeon per year. In fact, reports from UK centers that have expertise in pituitary tumors have observed that surgeons with a high workload have better outcomes. For a hospital treating a fixed population, a smaller number of surgeons produce better outcomes, and centers with only one neurosurgeon performing pituitary surgery have better outcomes than centers with several such surgeons [19, 20, 22, 29-31].

These observations have been endorsed by groups in other countries. A high workload, i.e., a high number of procedures performed each year, provides the neurosurgeon using the transsphenoidal approach with sufficient experience with respect to patient selection, operative techniques, and better outcomes, in addition to reducing the rate and severity of complications [9, 15, 21, 22, 24, 32, 33]. Finally, evaluation of efficacy must be based on outcome data [34,35].

Box 5. Experienced pituitary neurosurgeon for PTCOE: rationale and definition

1. Experienced pituitary neurosurgeons have better outcomes, and reduced rates of morbidity and mortality

2. The workload of a surgeon is based on the ratio between the number of operating surgeons and the size of the population served by the center

3. For a fixed number of inhabitants covered by the center, a reduced number of transsphenoidal pituitary surgeons results in better outcomes

4. The ideal number of transsphenoidal pituitary interventions for micro and macroadenomas for

an individual surgeon is debatable but should be approximately 50 per year

Assuming that expertise or excellence comes from the workload of a well-trained surgeon, and considering that most centers serve a fixed number of inhabitants, the solution proposed by several publications would be to have a limited number of surgeons devoted to pituitary tumors at each center, with a backup for emergency cases.

A single neurosurgeon situation, however, has several drawbacks. For example, the center will remain uncovered when the surgeon is absent, the training of new fellows would be difficult, and performing clinical research and communicating results could be impeded. For such reasons, one alternative could be to concentrate several neurosurgeons (two to four) at a given center that covers the transsphenoidal surgery needs of a whole region that ideally has 2.5 to 5 million inhabitants. Such a center would receive patients who were generated locally but also referred from endocrine units located at other hospitals in the region, i.e. "regionalization" for a pituitary center [11]. The regionalized center can receive patients already diagnosed, perform the intervention, and return the patient for follow up at the hospital or physician of origin. This process may be facilitated 
by implementation of electronic clinical registries working within a network. As the number of patients with pituitary diseases is not large, such an organization should be satisfactory in cost-benefit terms for health administrators, even considering the travel costs of the patient to the reference center.

Box 6. Organization of a neurosurgical center for a PTCOE

1. Expertise is based on workload, i.e., the quotient between a limited number of surgeons and a high number of patients

2. An alternative to reducing the number of surgeons performing transsphenoidal pituitary procedures is to expand the population covered

3. Having more than one neurosurgeon is convenient in terms of continuing access, training new fellows and scientific progress

4. An ideal reference center for a region may be formed by $2-4$ expert neurosurgeons performing transsphenoidal operations, including macro and micro adenomas serving a population of 2.5 to 5 million inhabitants, with a proportional increase in the work load

5. Such a regionalized unit can give surgical coverage to several external endocrine centers of excellence, and can return the patient to his/her local hospital for follow-up after surgery, according to follow up protocols

\section{Endocrine Units of Pituitary Tumors Centers of Excellence}

A PTCOE requires that the neurosurgery group works closely with an endocrinologist on an endocrine unit or a division that has a special emphasis on pituitary diseases. Obviously, pituitary diseases are not only tumors but encompass other pituitary pathologies and include the secondary effect of tumor treatment, such as central hyposecretion of TSH or LH/FSH, GH deficits, panhypopituitarism, as well as, Sheehan syndrome, diabetes insipidus, and so on, which are all managed by the same professionals [34-38]. At the end the endocrinologists need to serve a "generalist role" for a life long term and provide an holistic management for the patient. Only a combination of expert neurosurgeons working in liaison with expert neuroendocrinologists can provide the excellence of care needed to meet the definition of a PTCOE. This team will use advanced techniques of diagnoses and treatment, produce scientific reports, and present their results to scientific bodies and administrative authorities.

The mission of the endocrine component of the PTCOE includes suspecting pituitary disease, establishing the diagnosis, determining the optional treatment plan with neurosurgeons regarding surgical intervention, providing support for peri-operative care, and providing long term follow up management. Also document and manage any endocrine deficiencies across radiation procedures.

Defining expertise for an endocrinologist appears to be easier than for a neurosurgeon considering that the requiring workloadhas not be determined. It is evident, however, that an endocrinologist wishing to participate in a PTCOE needs to have received basic training in internal medicine and endocrinology though the residency stage, and then to perform postgraduate training for at least 12 months as part of a group of experts in pituitary disorders that has international stature.

The expert endocrinologist must have a through knowledge of the laboratory techiques for hormone analysis because diagnosis is based on the accurancy of such techiques. Analytical methods are complex and a true knowledge of reagents, calibrators and degree of standarization is mandatory. An adequate understanding of modern genetic techiques is also relevant for diagnosis. An expert endocrinologist must be able to rudimentary understanding of MRI studies to being able to read/interpret and review pituitary MRI studies, understand pituitary pathology, significance of positive immunostaining in absence of clinical signs- such as silent corticotroph tumors, and the importance (and limitations) of proliferative indices in regard to tumor growth.

To substantiate excellence, the endocrinologist should present results at scientific meetings and contribute to the advance of pituitary science. This can be supported by regular publications of peer-reviewed research articles, reviews, chapters in monographs or textbooks, participation in scientific or consensus meetings, as well as by regular participation in multi- center trials of novel pituitarydirected treatments strategies (Fig. 1).

Box 7. Experience of the endocrinologist working at a PTCOE

1. Specialty medical training in internal medicine or adequate alternatives and in endocrinology

2. Postgraduate training at a center with a unit dedicated to pituitary disorders

3. Working at a PTCOE with intense activity and a high workload

4. Presenting results at scientific events and to health administration bodies

5. Contributions to the advancement of pituitary science, and discovery in pituitary medicine

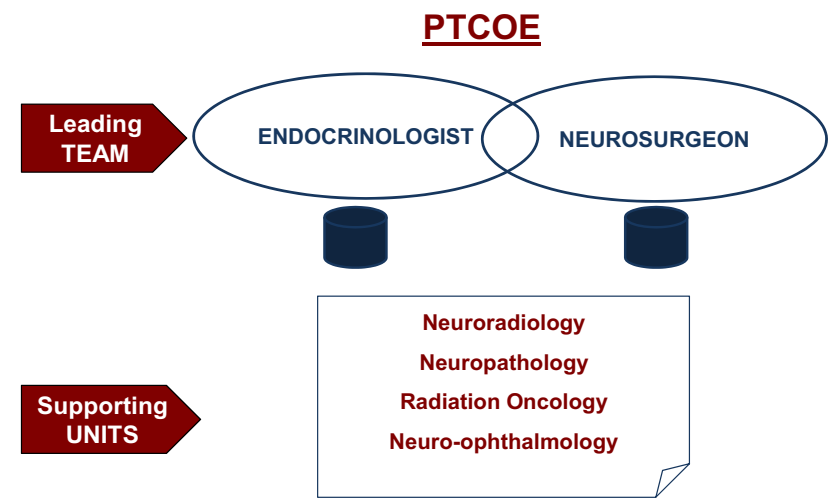

Fig. 1 General structure of a Pituitary Tumors Center of Excellence (PTCOE). The leading team composed by the endocrinology and by neurosurgery teams, and the main supportly units of other specialities 


\section{Supporting units for a PTCOE}

The close relationship of expert pituitary surgeons and pituitary endocrinologists requires the additional support of several specialties to provide a high-level standard of care. For imaging analysis, a group of well-trained neuroradiologists is ordinarily available in most centers and in all academic units. However in certain circumstances, familiarity with specific protocols to locate often small microadenomas in for example Cushing disease is of importance in a full PTCOE. For diagnosis, reports from the neuropathologist should include tumor immunocytochemistry to make full diagnosis and comments if the tumor exhibits unusually high proliferative potential as this may have bearing on subsequent management. There may be the need for close surgical collaboration between neurosurgery and otolaryngology in the execution of endonasal and other skull-base approaches. Neuro-ophthalmologists are required for the diagnosis and, in some cases, for follow-up. The contribution of such specialties is mandatory to provide a given PTCOE its necessary support [9, 39-47].

BOX 8. Units providing support to a PTCOE

\author{
1. Neuroradiology \\ 2. Neuropathology, including molecular diagnosis \\ 3. Radiation neuroncology \\ 4. Neurooncology \\ 5. Neuro-ophthalmology \\ 6. Specialized clinical and research nursing
}

\section{Expert Neuroradiology Unit}

At a minimum, high field magnetic resonance imaging (MRI) with at least $1.5 \mathrm{~T}$ field strength and high resolution depiction of the sellar region should be available for every patient with a pituitary tumor throughout the day. In addition, around the clock availability of thin-collimation computerized tomography is required for those who have contraindications to MRI. Orientation of the sections and labelling of the images should be standardized to enable reliable follow-up studies. The centre should have access to digital substraction angiograpy, and to expert selective bilateral venous sampling of the inferior petrosal sinus.

\section{Expert Neuropathology Unit}

Tumor Pathology is an essential aspect of the diagnosis, management and follow-up of patients with pituitary and related disorders. The pathologic diagnosis guides the optimal therapeutic strategy, and helps to determine the response to treatment and the prognosis for the patient.

The ideal arrangement for Pathology to be an integral part of the multidisciplinary PTCOE would be as follows:

BOX 9. Neuropathology unit

1. One or two experienced neuropathologists or endocrine pathologists who will have responsibility for the final diagnosis

2. Routine assessment of histology - mitoses, pleomorphism, giant cells, inclusions, inflammatory changes, stroma, hemorrhage, vascular features. Proliferative index Ki67. Routine stains

3. Routine pituitary hormone stains ACTH, prolactin, growth hormone, TSH, LH, FSH, and additionally in some cases alpha subunit, chromogranin, P53, hormone receptor stains, transcription factors

4. Tumor specimen banking

5. A standard report indicating a final diagnosis using the most current WHO guidelines and criteria with commentary on normal pituitary gland incorporated in the specimens

\section{Radiation Neuroncology Units of Excellence}

Radiation therapy is required to treat some pituitary tumors that are completely or partially resistant to medical treatment, or for surgical remnants from such tumors, patients who refuse or cannot undergosurgery,aggressive pituitary tumors or in pituitary cancers. In all of these cases, LINAC radiotherapy, stereotactic radiotherapy or radio-surgery, all of which are computer-assisted techniques performed by radiotherapists expert in the treatment of intracranial tumors, are necessary at a high level PTCOE [48-57].

Single fraction stererotactic radiosurgery (single dose) or fractionated stereotactic treatmentneed to be accessible from the center of excellence, to be utilized depending on patient and tumor related factors. Radiation neuroncologists treating patients with pituitary adenomas must have an indepth knowledge of the tolerance of the optic system, cranial nerves of the cavernous sinus, temporal lobes and the normal pituitary gland. They should also understand the temporal relationship between the delivery of radiation and the interval for development of such complications as hypopituitarism. Radiation oncologists should work in close cooperation with the neuroendocrinologists and neurosurgeons.This should also allow selected radiotherapists to gain enough experience in the treatment of pituitary tumors.

In some national Health Systems, radiation neurooncology units are not located in every high level hospital but at specific centers. In such situations close relationship between radiotherapists and neuroendocrinologists must be guaranteed.

All of these different specialties working in a team with neurosurgeons and expert neuroendocrinologists form the 
Fig. 2 Working flow chart of a given PTCOE. The Unit of Excellence benefits of working in collaboration with external endocrine units that may be located in other centers or hospitals. After surgery patients return to their original units. This allows for a networking method of patient care

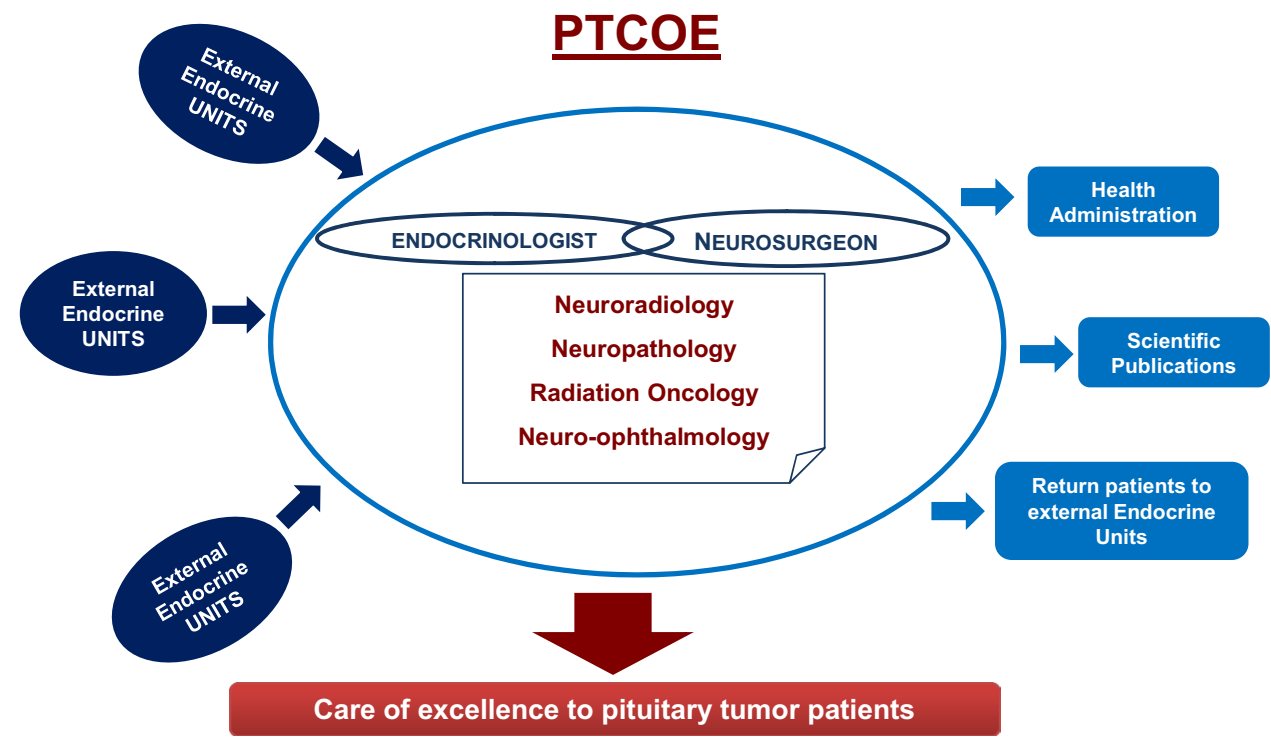

core of a PTCOE. The internal architecture of the unit and facilities should allow intense collaborative and interdisciplinary functioning. In some national Health Systems there are regional endocrine centers of high quality that may interact with a PTCOE. A bidirectional flux the patients would guarantee the best care for patients suffering of pituitary pathologies (Fig. 2).

\section{Conclusions}

In the last few decades, a considerable body of evidence supports the concept that patients with pituitary tumors would receive the best care from units of excellence composed of expert neurosurgeons performing pituitary surgery by transsphenoidal and other approaches, plus experienced neuroendocrinologists devoted to these types of tumors. These experts, working in liaison with supporting units, would form a center of excellence for pituitary tumors (PTCOE). Such a center would be the optimal organization for patients, the most cost-effective for health administrators, and a more suitable structure to allow for derivation and presentation of results, and advancement of pituitary science. The present document is intended to provide the basis for such a PTCOE structure.

Collaborators for The Pituitary Society, Expert Group on Pituitary Tumors Alin Abreu Lomba (Endocrinologist of skull base centro medico Imbanaco, Cali, Colombia, alin.abreu@imbanaco.com.co); Julio Abucham (Neuroendocrinology Unit, Escola Paulista de Medicina da Universidade Federal de São Paulo, São Paulo, Brazil, julioabucham@uol.com.br); Cristina Alvarez-Escola (Neuroendocrinology Unit, University Hospital La Paz, Madrid, Spain, escola.cristina@ gmail.com); Ariel L Barkan (Division of Endocrinology, University of Michigan Health System, Ann Arbor, MI, USA, abarkan@umich.edu);
Albert Beckers (Department of Endocrinology, CHU de Liège, Domaine Universitaire du Sart-Tilman, Liege, Belgium, albert.beckers@chu.ulg.ac.be); Anat Ben-Shlomo (Pituitary Center, Division of Endocrinology, Diabetes \& Metabolism, Cedars-Sinai Medical Center, Los Angeles, CA, USA, Anat.BenShlomo@cshs.org); Ignacio Bernabeu (Endocrinology Division, Complejo Hospitalario Universitario de Santiago de Compostela (CHUS)-SERGAS, Santiago de Compostela, Spain, ignacio.bernabeu.moron@sergas.es); Martin Bidlingmaier (Endocrine Laboratory, Medizinische Klinik und Polklinik IV, Klinikum der Universität Munich, Germany, martin.bidlingmaier@med. uni-muenchen.de); Nienke Biermasz (Division of Endocrinology, Department of Internal Medicine, Leiden University Medical Center, Leiden, The Netherlands, n.r.biermasz@lumc.nl); Beverly Biller (Neuroendocrine Unit, Massachusetts General Hospital, Harvard Medical School, Boston, MA, USA, bbiller@ partners.org); Cesar Luiz Boguszewski (SEMPR, Endocrine Division, Department of Internal Medicine, Federal University of Parana, Curitiba, Brazil cesar.boguszewski@ hc.ufpr.br); Marek Bolanowski (Department of Endocrinology, Diabetes and Isotope Therapy, Wroclaw Medical University, Wroclaw, Poland, marek.bolanowski@umed.wroc.pl); Jens Bollerslev (Section of Specialized Endocrinology, Department of Endocrinology, Oslo University Hospital, Rikshospitalet, Nydalen, Faculty of Medicine, University of Oslo, Oslo, Norway, Jens.bollerslev@ medisin.uio.no); Vivien Bonert (Department of Medicine, Cedars-Sinai Medical Center, Los Angeles, CA, USA, Vivien.Bonert@ cshs.org); Marcello Bronstein (Neuroendocrine Unit, Division of Endocrinology and Metabolism, Hospital das Clinicas, University of Sao Paulo, Sao Paulo, Brazil, mdbronstein@uol.com.br); Oscar D Bruno (University of Buenos Aires, Buenos Aires, Argentina, divendhcli@intramed.net); Michael Buchfelder (Department of Neurosurgery, University Hospital Erlangen, Erlangen, Germany, michael.buchfelder@uk-erlangen.de); John D Carmichael (Keck School of Medicine of the University of Southern California, Los Angeles, CA, USA, John.Carmichael@med.usc.edu); Philippe Caron (Department of Endocrinology and Metabolic Diseases, CHU Larrey, Toulouse, France, caron.p@ chu-toulouse.fr); Philippe Chanson (Endocrinology and Reproductive Diseases, Hôpitaux Universitaires Paris-Sud Hôpital de Bicêtre and Université Paris-Sud IDEX Univ Paris-Saclay, Paris, France, philippe.chanson@bct.aphp. fr); Felipe F Casanueva (Division of Endocrinology, Santiago de Compostela University, Santiago de Compostela, Spain, endocrine@usc. es); Richard N Clayton (Department of Medicine, Keele University, Stoke on Trent, UK, r.n.clayton@keele.ac.uk); Annamaria Colao 
(Division of Endocrinologia, Universita' Federico II di Napoli, Naples, Italy, colao@unina.it); Fernando Cordido (Endocrinology and Nutrition, University of A Coruña, A Coruña University Hospital, A Coruña, Spain, fernando.cordido.carballido@sergas.es); Laura De Marinis (Universita' Cattolica del Sacro Cuore Roma, Pituitary Unit, Fondazione Policlinico A. Gemelli, Rome, Italy, laurademarinis@yahoo.it); Rudolf Fahlbusch (International Neuroscience Institute, Hannover, Germany, neurochirurg-prof-fahlbusch@web.de); Maria Fleseriu (Medicine and Neurological Surgery, Pituitary Center, Oregon Health \& Science University, Portland, OR, USA, fleseriu@ ohsu.edu); Anna Maria Formenti (Department of Molecular and Translational Medicine, University of Brescia, Brescia, Italy, annaformenti@live.it); Pamela U Freda (Division of Medicine, Columbia University, New York, NY, USA, puf1@cumc.columbia.edu); Hidenori Fukuoka (Department of Internal Medicine, Division of Diabetes and Endocrinology, Kobe University Hospital, 7-5-2 Kusunoki-cho, Chuo-ku, Kobe, Japan, fukuokah@med.kobe-u.ac.jp); Ezio Ghigo (Division of Endocrinology, Diabetes and Metabolism, University of Turin, Turin, Italy, ezio. ghigo@unito.it); Andrea Giustina (Division of Endocrinology, San Raffaele University Hospital, Milan, Italy, giustina.andrea@hsr.it); Yona Greenman (Institute of Endocrinology and Metabolism, Tel AvivSourasky Medical Center and Sackler Faculty of Medicine, Tel Aviv University, Tel Avivi, Israel, yonagr@tlvmc.gov.il); Elena Grineva (Endocrinology Institute, Federal Almazov North-West Medical Reseach Centre, Moscow, Russia, grineva_e@ mail.ru); Ashley Grossman (Division of Endocrinology, University of Oxford, Royal Free London Hospital, Barts and the London School of Medicine, Oxford, UK, ashley.grossman@ocdem.ox.ac.uk); Mark Gurnell (University of Cambridge \& Addenbrooke's Hospital, Metabolic Research Laboratories, Wellcome Trust-MRC Institute of Metabolic Science, Cambridge, UK, mg299@medschl.cam.ac.uk); Anthony Heaney (Department of Medicine, Cedars-Sinai Medical Center, Los Angeles, CA, USA, AHeaney@mednet.ucla.edu); Andrew R Hoffman (Department of Medicine, Stanford University School of Medicine, Endocrinology Section, VA Palo Alto Health Care System, CA, USA, glands@stanford.edu); Irena Ilovayskaya (Department of Therapeutical Endocrinology, Moscow Regional Research and Clinical Institute (MONIKI), Moscow, Russia, irena.ilov@yandex.ru); Gudmundur Johannsson (Department of Endocrinology Institute of Medicine, Sahlgrenska Academy, University of Gothenburg, Gothenburg, Sweden, gudmundur.johannsson@medic.gu.se); Pinar Kadioglu (University of Istanbul, Cerrahpasa Medical Faculty, Istanbul, Turkey, kadioglup@yahoo.com); Niki Karavitaki (Centre for Endocrinology, Diabetes and Metabolism, Institute of Metabolism and Systems Research, University of Birmingham, Birmingham, UK, nikikara@hotmail.com); Laurence Katznelson (Departments of Medicine and Neurosurgery, Stanford University School of Medicine 875 Blake Wilbur Dr MC 5826, Stanford, CA, USA, 1katznelson@stanford.edu); Fahrettin Kelestimur (Department of Endocrinology, Erciyes University Medical School, Kayseri, Turkey, fktimur2001@yahoo.co.uk); Daniel F Kelly (Pacific Brain Tumor Center \& Pituitary Disorders Center, John Wayne Cancer Institute at Providence Saint John's Health Center, Santa Monica, CA, USA, kellyd@jwci.org); Anne Klibanski (Neuroendocrine Unit, Massachusetts General Hospital, Boston, MA, USA, aklibanski@partners.org); Ken Ho (Centres for Health Research and the Department of Endocrinology, Princess Alexandra Hospital, University of Queensland, Queensland, Australia, Ken_Ho@ health.qld.gov.au); Michal Krsek (Department of Medicine and Centre for Rare Endocrine Diseases, University Hospital Kralovske Vinohrady, 3rd Faculty of Medicine of the Charles University, Czech Republic, Prague, michal.krsek@fnkv.cz); Andre Lacroix (Département de Médecine, Service d'Endocrinologie, Laboratoire de Pathophysiologie Endocrinienne, Centre de Recherche du CHUM (CRCHUM), Pavillon Jeanne-Mance, Centre hospitalier de l'Université de Montréal (CHUM), Montréal, Canada, andre.lacroix@ umontreal. ca); Edward R Laws (Department of Neurosurgery, Brigham \& Women’s Hospital, Boston, MA, USA, elaws@ partners.org); Jay Steven
Loeffler (Department of Radiation Oncology, Massachusetts General Hospital, Boston, MA, USA, jloeffler@mgh.harvard.edu); Marco Losa (Department Of Neurosurgery, Istituto Scientifico San Raffaele, Milan, Italy, losa.marco@ hsr.it); Jens Otto Jørgensen (Department of Endocrinology and Internal Medicine, Aarhus University Hospital Aarhus, Denmark, joj@clin.au.dk); Anton Luger (Division of Endocrinology and Metabolism, Department of Medicine III, Medical University and General Hospital of Vienna, Vienna, Austria, anton.luger@ meduniwien.ac.at); Susana Mallea-Gil (Division of Endocrinology, Hospital Carlos G. Durand, Buenos Aires, Argentina, smalleagil@gmail.com); Adam Mamelak (Department of Neurosurgery, Cedars-Sinai Medical Center, Los Angeles, CA. USA, adam.Mamelak@cshs.org); Gherardo Mazziotti (Endocrinology Unity, Mantova Hospital, Mantova, Italy, gherardo.mazziotti@asst-mantova.it); Ann McCormack (St Vincent's Hospital, Garvan Institute of Medical Research, Sydney, Australia, a.mccormack@garvan.org.au); Shlomo Melmed (Cedars-Sinai Medical Center, Los Angeles, CA, USA, melmed@cshs.org); Moises Mercado (Division of Medicine, National Autonomous University of Mexico, Experimental Endocrinology Unit, Centro Médico Nacional, Siglo XXI, IMSS, Mexico City, Mexico, moises.mercado@endocrinologia. org.mx); Pietro Mortini (Department of Neurosurgery, San Raffaele University Health Institute, Milan, Italy, mortini.pietro@ hsr.it); Sebastian Neggers (Section of Endocrinology, Department of Medicine, Erasmus University Medical Centre, Rotterdam, The Netherlands, s.neggers@erasmusmc.nl); Guang Ning (Chinese National Clinical Research, Center for Endocrine and Metabolic Diseases, Shanghai Jiaotong University School of Medicine, Shangai, China, gning@ sibs. ac.cn); Nelson M Oyesiku (Division of Neurosurgery and Medicine Endocrinology, Emory Pituitary Center, Dept. of Neurosurgery, Emory University School of Medicine, Atlanta, USA, noyesik@emory.edu); Vera Popovic (Medical School University of Belgrade, Belgrade, Serbia, popver@gmail.com); Milan Petakov (Department for Neuroendocrinology, Clinic for Endocrinology, Clinical Center of Serbia, Belgrade, Serbia, mpetakov@EUnet.rs); Stephan Petersenn (ENDOC Center for Endocrine Tumors, Hamburg, Germany, stephan.petersenn@ endoc-med.de); Misa Pfeifer (Janssen Scientific Affairs, LLC, Titusville, USA, mpfeifer@its.jnj.com); Antonio Pico (Department of Endocrinology \& Metabolism. Hospital General Universitario de Alicante, University Miguel Hernandez, Alicante, Spain, antonio.pico@umh.es); Manuel Puig Domingo (Dpt of Medicine, Universitat Autonoma de Barcelona, Germans Trias i Pujol HealthSciences Institute, Badalona, Spain, mpuigd@igtp.cat); Gérald Raverot (Centre de Référence Maladies Rares Hypophysaires HYPO, Hospices Civils de Lyon, Inserm U1052, Lyon, France, gerald.raverot@chu-lyon.fr); Martin Reincke (Medizinische Klinik IV. Klinikum der LMU, Munich, Germay, martin. reincke@med.uni-muenchen.de); Monica Roberto Gadelha (Neuroendocrinology Research Center/Endocrinology Section, Medical School and Hospital Universitário Fraga Filho, Universidade Federal do Rio de Janeiro; Rio de Janeiro, Brazil, mgadelha@ @ucff.ufrj.br); Roberto Salvatori (Division of Medicine and Neurosurgery. Pituitary Center, Division of Endocrinology, Diabetes and Metabolism, Baltimore, USA, salvator@jhmi.edu); Susan L Samson (Division of Diabetes, Endocrinology and Metabolism. Pituitary Center, Baylor College of Medicine, Houston, TX, USA, ssamson@bcm.edu); Akira Shimatsu (National Hospital Organization Kyoto Medical Center, Kyoto, Japan, ashimats@ kyotolan.hosp.go.jp); Ilan Shimon (Endocrine Institute, Rabin Medical center, Beilinson Hospital, Petah-Tikva, Israel, Ilanshi@clalit.org.il); Paul Stewart (University of Leeds. Worsley Building, Clarendon Way, Leeds, UK, P.M.Stewart@ leeds.ac.uk); Christian Strasburger (Department of Medicine for Endocrinology Diabetes and Nutritional Medicine, Charité Universitätsmedizin, Berlin, Germany, Christian.Strasburger@charite.de); Brooke Swearingen (Neuroendocrine Clinical Center, Massachusetts General Hospital, Boston, USA, BSWEARINGEN@mgh.harvard.edu); Peter Trainer (Department of Endocrinology, The Christie NHS Foundation Trust, Manchester, UK, peter.trainer@ manchester.ac.uk); Nicholas A Tritos (Massachusetts General Hospital, 
Neuroendocrine and Pituitary Clinical Center, Zero Emerson Place, Boston, MA, USA, NTRITOS@mgh.harvard.edu); Stylianos Tsagarakis (Department of Endocrinology, Diabetes and Metabolism, Evangelismos Hospital, Athens, Greece, stsagara@otenet.gr); AJ van der Lely (Department of Internal Medicine, Section of Endocrinology, Erasmus University MC, Rotterdam, The Netherlands, a.vanderlelij@ erasmusmc.nl); Lucio Vilar (Department of Internal Medicine, Endocrinology Division, Hospital das Clínicas / Federal University of Pernambuco, Pernambuco, Brazil, lvilarf@gmail.com); Rocio Villar-Taibo (Endocrinology and Nutrition Division, Clinical University Hospital of Santiago de Compostela, Santiago de Compostela, Spain, rotaibo22@gmail.com); John Wass (Oxford Centre for Diabetes, Endocrinology and Metabolism, Churchill Hospital, Oxford, UK, john. wass@ nhs.net); Maria Chiara Zatelli (Section of Endocrinology \& Internal Medicine, Department of Medical Sciences, University of Ferrara, Ferrara, Italy, ztlmch@unife.it).

\section{Compliance with ethical standards}

Conflict of interest Felipe F Casanueva is consultant and lecture of fees for Pronokal, NovoNordisk and Orexigen and, member of Board of Directors Pituitary Society; Ariel L Barkan is research grant for Novartis and consultant for Pfizer, Ipsen and Novartis; Anne Klibanski is research grant for Ipsen, consultant for Chiasma and member of Scientific Board of Directors for Crinetics and Pituitary Society; Shlomo Melmed is research grant for Pfizer and Ipsen, and consultant for Chiasma, Novartis, Stonebridge, Ionis, Ono, Midatech, Dauntless, Reset and member of Board of Directors Pituitary Society; Andrea Giustina is consultant for Ipsen, Novartis and Pfizer, and member of Board of Directors Pituitary Society. The other authors (Michael Buchfelder, Edward R. Laws, Jay S Loeffler, Pietro Mortini and John Wass) declare they have no conflict of interest.

Informed consent This article does not contain any studies with human participants or animals performed by any of the authors.

Open Access This article is distributed under the terms of the Creative Commons Attribution 4.0 International License (http://creativecommons.org/licenses/by/4.0/), which permits unrestricted use, distribution, and reproduction in any medium, provided you give appropriate credit to the original author(s) and the source, provide a link to the Creative Commons license, and indicate if changes were made.

\section{References}

1. Daly AF, Tichomirowa MA, Beckers A (2009) The epidemiology and genetics of pituitary adenomas. Best Pract Res Clin Endocrinol Metab 23:543-554

2. Fernandez A, Karavitaki N, Wass JA (2010) Prevalence of pituitary adenomas: a community-based, cross-sectional study in Banbury (Oxfordshire, UK). Clin Endocrinol 72:377-82

3. Giustina A, Chanson P, Kleinberg D et al (2014) Expert consensus document: A consensus on the medical treatment of acromegaly. Nat Rev Endocrinol 10:243-248

4. Melmed S, Casanueva FF, Cavagnini F et al (2002) Guidelines for acromegaly management. J Clin Endocrinol Metab 87:4054-4058

5. Barker FG, Klibanski A, Swearingen B (2003) Transsphenoidal surgery for pituitary tumors in the United States, 1996-2000: mortalitiy, morbidity, and the effects of hospital and surgeon volume. J Clin Endocrinol Metab 88:4709-4719
6. Weisman CS, Squires GL (2000) Women's health centers: are the national centers of excellence in women's health a new model? Womens Health Issues 10:248-255

7. Champion JK, Pories WJ (2005) Centers of excellence for bariatric surgery. Surg Obes Relat Dis 1:148-151

8. Pratt GM, McLees B, Pories WJ (2006) The ASBS bariatric surgery centers of excellence program: a blueprint for quality improvement. Surg Obes Relat Dis 2:497-503

9. McLaughlin N, Laws ER, Oyesiku NM et al (2012) Pituitary centers of excellence. Neurosurgery 71:916-924

10. Swearingen B (2012) Update on pituitary surgery. J Clin Endocrinol Metab 97:1073-1081

11. Luft HS, Bunker JP, Enthoven AC (1979) Should operations be regionalized? The empirical relation between surgical volume and mortality. N Engl J Med 301:1364-1369

12. Schwartz TH (2013) A role for centers of excellence in transsphenoidal surgery. World Neurosurgery 80:270-271

13. Mercado M, Gonzalez B, Vargas G et al (2014) Successful mortality reduction and control of comorbidities in patients with acromegaly followed at a highly specialized multidisciplinary clinic. J Clin Endocrinol Metab 99:4438-4446

14. Faustini-Fustini M, Pasquini E, Zoli M et al (2013) Pituitary centers of excellence. Neurosurgery 73:557-558

15. Knutzen R (2014) Pituitary centers of excellence: for patients it is life or death. Neurosurgery 74:143-144

16. Koc K, Anik I, Ozdamar D et al (2006) The learning curve in endoscopic pituitary surgery and our experience. Neurosurg Rev, $29,298-305$

17. Snyderman C, Kassam A, Carrau R et al (2007) Acquisition of surgical skills for endonasal skull base surgery: a training program. Laryngoscope 117:699-705

18. Lee TT, Klose JL, Congress of Neurological Surgeons Education Committee (1999) Survey on neurosurgery subspecialty fellowship training. Surg Neurol 52:641-644

19. Yamada S, Aiba T, Takada K et al (1996) Retrospective analysis of long-term surgical results in acromegaly: preoperative and postoperative factors predicting outcome. Clin Endocrinol 45:291-298

20. Gittoes NJ, Sheppard MC, Johnson AP et al (1999) Outcome of surgery for acromegaly - the experience of a dedicated pituitary surgeon. Q J Med 92:741-745

21. Erturk E, Tuncel E, Kiyici S et al (2005) Outcome of surgery for acromegaly performed by different surgeons: importance of surgical experience. Pituitary 8:93-97

22. Bates PR, Carson MN, Trainer PJ et al (2008) Wide variation in surgical outcomes for acromegaly in the UK. Clin Endocrinol 68:136-142

23. Shahlalie K, McLaughlin N, Kassam AB et al (2010) The role of outcomes data for assessing the expertise of a pituitary surgeon. Curr Opin Endocrinol Diabetes Obes 17:369-376

24. Ciric I, Ragin A, Baumgartner C et al (1997) Complications of transsphenoidal surgery: results of a national survey, review of the literature, and personal experience. Neurosurgery 40:225-236

25. Fatemi N, Dusick JR, de Paiva Neto MA et al (2008) The endonasal microscopic approach for pituitary adenomas and other parasellar tumors: a 10-year experience. Neurosurgery 63:244-256

26. Laws ER, Fode NC, Redmond MJ (1985) Transsphenoidal surgery following unsuccessful prior therapy. An assessment of benefits and risks in 158 patients. J Neurosurg 63:823-829

27. Mattozo CA, Dusick JR, Esposito F et al (2006) Suboptimal sphenoid and sellar exposure: a consistent finding in patients treated with repeat transsphenoidal surgery for residual endocrine-inactive macroadenomas. Neurosurgery 58:857-865

28. Saeki N, Yamaura A, Numata T et al (1999) Transsphenoidal reoperations for removal of pituitary adenomas: rhinological management and timing of reoperation. J Clin Neurosci 6:385-388 
29. Ahmed S, Elsheikh M, Stratton IM et al (1999) Outcome of transsphenoidal surgery for acromegaly and its relationship to surgical experience. Clin Endocrinol 50:561-567

30. Birkmeyer JD, Siewers AE, Finlayson EV et al (2002) Hospital volume and surgical mortality in the United States. N Engl J Med 346:1128-1137

31. Barker FG, Curry WT Jr, Carter BS (2005) Surgery for primary supratentorial brain tumors in the United States, 1988 to 2000: the effect of provider case load and centralization of care. Neuro Oncol 7:49-63

32. Jane JA, Laws ER (2001) The surgical management of pituitary adenomas in a series of 3,093 patients. J Am Coll Surg 193:651-659

33. Wilson CB (1997) Surgical management of pituitary tumors. J Clin Endocrinol Metab 82:2381-2385

34. Powell M, Grossman A (2016) Quality indicators in pituitary surgery: a need for reliable and valid assessment. What should be measured? Clin Endo 84:485-488

35. Ammirati M, Wei L, Ciric I (2013) Short-term outcome of endoscopic versus microscopic pituitary adenoma surgery: a systematic review and meta-analysis. J Neurol Neurosurg Psychiatry 84:843-849

36. Ascoli P, Cavagnini F et al (2006) Hypopituitarism Pituitary 9:335-342

37. Casanueva FF, Molitch ME, Schlechte JA et al (2006) Guidelines of the Pituitary Society for the diagnosis and management of prolactinomas. Clin Endocrinol 65:265-273

38. Maffezzoni F, Formenti AM, Mazziotti G et al (2016) Current and future medical treatments for patients with acromegaly. Expert Opin Pharmacother 17:1631-1642

39. Evans CO, Young AN, Brown MR et al (2001) Novel patterns of gene expression in pituitary adenomas identifiied by complementary deoxyribonucleic acid microarryas and quantitative RT-PCR. J Clin Endocrinol Metab 86:3097-3107

40. Kontogeorgos G (2005) Classification and pathology of pituitary tumors. Endocr 28:27-35

41. Moreno CS, Evans CO, Zhan X et al (2005) Novel molecular signaling and classification in human clinically non-functional pituitary adenomas identified by gene expression profiling and proteomics analyses. Cancer Res 65:10214-10222

42. Saeger W, Lüdecke DK, Buchfelder M et al (2007) Pathohistological classification of pituitary tumors: 10 years of experience with the German Pituitary Tumor Registry. Eur J Endocrinol 156:203-216

43. O'Malley BW, Grady MS, Gabel BC et al (2008) Comparison of endoscopic and microscopic removal of pituitary adenomas: single-surgeon experience and the learning curve. Neurosurg Focus 25:E10

44. Gejman R, Swearingen B, Hedley-Whyte ET et al (2008) Role of $\mathrm{Ki}-67$ proliferation index and $\mathrm{p} 53$ expression in predicting progression of pituitary adenomas. Hum Pathol 39:758-766

45. Galland F, Lacroix L, Saulnier P et al (2010) Differential gene expression profiles of invasive and non-invasive non-functioning pituitary adenomas base on microarray analysis. Endoc Relat Cancer 17:361-371

46. Bush ZM, Longtine JA, Cunningham T et al (2010) Temozolomide treatment for aggressive ppituitary tumors: correlation of clinical outcome with $\mathrm{O}(6)$-methylguanine methyltransferase (MGMT) promoter methylation and expression. J Clin Endocrinol Metab 95:280-290

47. Salehi F, Agur A, Scheithauer BW et al (2010) Biomarkers of pituitary neoplasms: a review (Part II). Neurosurgery 67:1790-1798

48. Sheehan JM, Vance ML, Sheehan JP et al (2000) Radiosurgery for Cushing's disease after failed transsphenoidal surgery. J Neurosrug 93:738-745

49. Jane JA, Vance ML, Woodburn CJ et al (2003) Stereotactic radiosurgery for hypersecreting pituitary tumors: part of a multimodality approach. Neurosurg Focus, 14:e12

50. Behbehani RS, McElveen T, Sergott RC et al (2005) Frationated stereotactic radiotherapy for parasellar meningiomas: a preliminary report of visual outcomes. Br J Ophthalmol 89:130-133

51. Melmed S, Colao A, Barkan A et al (2009) Guidelines for acromegaly management: an update. J Clin Endocrinol Metab 94:1509-1517

52. Jensen R, O'Rourke D, Warnick R et al (2006) Resident training in neurosurgical oncology: results of the survey of North American training programs by the AANS/CNS Section on Tumors. J Neurooncol 77:241-246

53. McLaughlin N, Kassam AB, Prevedello DM et al (2011) Management of Cushing's disease after failed surgery-a review. Can J Neurol Sci 38:12-21

54. Loeffler JS, Shih HS (2011) Radiation therapy in the management of pituitary adenomas. J Clin Endocrinol Metab 96:1992-2003

55. Shrieve DC,Loeffler JS (2010) Human radiation injury. Lippincott Williams and Wilkins, Philadelphia, p 560

56. Mortini P, Losa M, Barzaghi R et al (2005) Results of transsphenoidal surgery in a large series of patients with pituitary adenoma. Neurosurgery 56:1222-1233

57. Burman P, van Beek AP, Biller M.K.B. et al (2017) Radiotherapy, especially at young age, increases the risk for de novo brain tumors in patients treated for pituitary/sellar lesions. J Clin Endocrinol Metab 102:1051-1058 